\title{
Observations of Pre-Stellar Cores
}

\author{
M. Tafalla \\ Observatorio Astronómico Nacional, Alfonso XII 3, E-28014 Madrid, Spain \\ email: m.tafalla@oan.es
}

\begin{abstract}
Our understanding of the physical and chemical structure of pre-stellar cores, the simplest star-forming sites, has significantly improved since the last IAU Symposium on Astrochemistry (South Korea, 1999). Research done over these years has revealed that major molecular species like CO and CS systematically deplete onto dust grains in the interior of pre-stellar cores, while species like $\mathrm{N}_{2} \mathrm{H}^{+}$and $\mathrm{NH}_{3}$ survive in the gas phase and can usually be detected toward the core centers. Such a selective behavior of molecular species gives rise to a differentiated (onion-like) chemical composition, and manifests itself in molecular maps as a dichotomy between centrally peaked and ring-shaped distributions. From the point of view of star-formation studies, the identification of molecular inhomogeneities in cores helps to resolve past discrepancies between observations made using different tracers, and brings the possibility of self-consistent modelling of the core internal structure. Here I present recent work on determining the physical and chemical structure of two pre-stellar cores, L1498 and L1517B, using observations in a large number of molecules and Monte Carlo radiative transfer analysis. These two cores are typical examples of the pre-stellar core population, and their chemical composition is characterized by the presence of large 'freeze out holes' in most molecular species. In contrast with these chemically processed objects, a new population of chemically young cores has begun to emerge. The characteristics of its most extreme representative, L1521E, are briefly reviewed.
\end{abstract}

Keywords. astrochemistry — ISM: clouds - ISM: molecules — molecular processes — radiative transfer - radio lines: ISM — stars: formation

\section{Pre-stellar Cores as Star-Forming Sites}

Pre-stellar (or starless) cores are the simplest star-forming sites. They are isolated, lie nearby, and form one star (or one binary) at the time; they closely resemble the theorist's ideal of a star forming region. When observed in a molecular tracer like ammonia, a prestellar core appears as a centrally concentrated structure containing one or a few solar masses of material and having a typical size of about 0.1 pc (see Figure 1 and Benson \& Myers 1989 for further global properties of cores).

Pre-stellar cores are the dominant star-forming sites in nearby molecular clouds like Taurus-Auriga, where stars like our Sun are currently forming in the so-called "isolated mode" (e.g., Shu, Adams, \& Lizano 1987). They are not, however, the dominant starforming regions of our galaxy, as most stars in the Milky Way have formed in groups (e.g., Adams \& Myers 2001), and therefore must result from the collapse of more complex gas structures. Still, star formation in isolated pre-stellar cores seems to involve most of the physical elements that we associate with the birth of a typical low-mass star, like gravitational infall, disk formation, and bipolar outflow ejection. All these elements, in fact, were first identified in stars forming in isolated cores, and they can be studied with great detail in these simple environments.

The above reasons of simplicity make pre-stellar cores ideal sites to study the initial conditions of star-formation (e.g., Ward-Thompson et al. 1999). Cores are also the most promising places to test the different competing models of star formation, in particular the (fast) turbulence driven scenario (Mac Low \& Klessen 2004) and the (slow) 


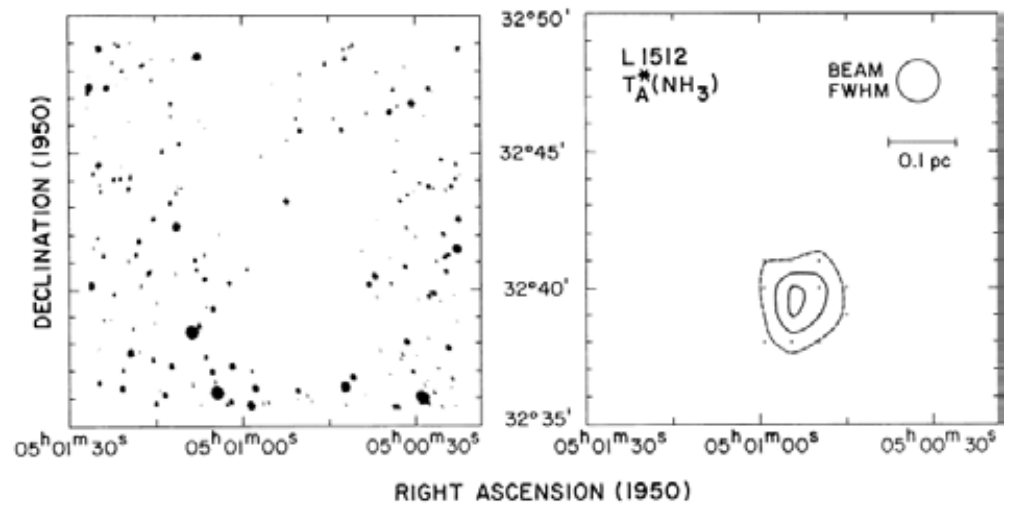

Figure 1. A representative pre-stellar core, L1512. Left: Optical image from the Palomar Sky Survey, where L1512 appears as a patch of obscuration against the background of stars. Right: Map of the $\mathrm{NH}_{3}$ emission toward the same region showing L1512 as a centrally concentrated (slightly resolved) object. Figure from Benson \& Myers (1989).

magnetically-mediated star formation models (Shu et al. 1987; Mouschovias \& Ciolek 1999). By determining how pre-stellar cores contract out of the more diffuse ambient cloud and by what mechanism cores lose their gravitational support and start collapsing to form a star, we may be able to observationally distinguish between these two models.

\section{Cores as Laboratories of ISM Chemistry}

Pre-stellar cores are also some of the simplest chemical laboratories in the interstellar medium (ISM) because of their isolation, approximately spherical geometry, and low $(10 \mathrm{~K})$, close to constant gas temperature. Despite this simplicity, however, pre-stellar cores are not chemically homogeneous, and the ignorance of this fact has caused, in the past, serious difficulties when attempting to derive the core internal structure from observations. Early warnings of chemical inhomogeneities were the striking discrepancies found when mapping cores using different molecular tracers. Classic examples of this problem are the observations made using $\mathrm{NH}_{3}$ and $\mathrm{CS}$, two molecular species expected to trace similar gas conditions. As found by Zhou et al. (1989), maps made in $\mathrm{NH}_{3}$ and CS present systematically different sizes $\left(\mathrm{NH}_{3}\right.$ maps are a factor of 2 smaller), different shapes, and often different peak positions. Resolution effects or radiative transfer complexities were soon found insufficient to explain these discrepancies.

A hint of a solution to the tracer discrepancies comes from the recent realization that molecules such as CO and CS are systematically depleted at the centers of cores due to the freeze out onto cold dust grains (Kuiper et al. 1996; Willacy et al. 1998; Kramer et al. 1999; Caselli et al. 1999). The identification of molecular freeze out in cores (long expected from theoretical grounds, see Watson \& Salpeter 1972) has renewed the interest in the study of dense-core chemistry, and brings with it the promise of resolving the old tracer discrepancies with relatively simple chemical processes. From the point of view of star-formation studies, the identification of freeze out in some molecules is forcing a reevaluation of the behavior of the different dense gas tracers under typical core conditions, as we depend on them to infer basic core properties like gas temperature and kinematics. Determining the chemical structure of cores has therefore become a necessary step in our attempt to understand how stars are born. 

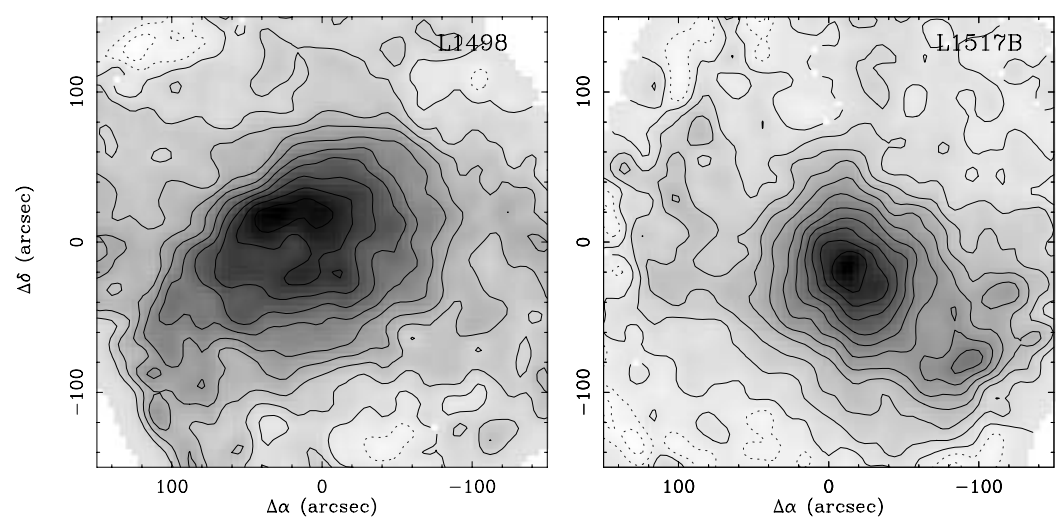

Figure 2. $1.2 \mathrm{~mm}$ continuum maps of L1498 (left) and L1517B (right). Note the central concentration and close-to-round shape of the emission. First contour and contour spacing are 2 mJy/11"-beam.

\section{A Molecular Survey of L1498 and L1517B}

To understand the chemical behavior of dense-gas tracers in star-forming regions, we have carried out a systematic molecular line survey of two pre-stellar cores in the TaurusAuriga cloud complex, L1498 and L1517B (Tafalla, Myers, Caselli, \& Walmsley 2004; Tafalla et al. 2005, in preparation). We selected these two cores (Fig. 2) for being isolated, close to round, and otherwise typical cores of their surrounding cloud, and we have observed them in the $1.2 \mathrm{~mm}$ continuum and in a large number of molecular lines from thirteen different species using the IRAM 30m, Effelsberg $100 \mathrm{~m}$, and FCRAO $14 \mathrm{~m}$ radio telescopes.

The goal of this project is to model self-consistently all the observed emission in order to determine how the different species trace the core interior, and to provide a high quality set of molecular abundances for testing chemical models. We can divide the analysis of the observations in two steps. First, we determine the physical parameters of the cores by modelling their distributions of density, temperature, and gas kinematics assuming that the cores are spherically symmetric. Once these parameters have been fixed, the cores can be seen as laboratories of known physical properties, and the abundances of the different molecular species can be derived directly by fitting their observed emission.

\subsection{Physical Structure of L1498 and L1517B}

To derive the density profiles of L1498 and L1517B we rely on the dust continuum emission, as this is the most unbiased tracer of the core column density. We assume a $1.2 \mathrm{~mm}$ dust emissivity of $0.005 \mathrm{~cm}^{2} \mathrm{~g}^{-1}$ and a dust temperature of $10 \mathrm{~K}$, and we note that these parameters are the largest source of uncertainty of the whole analysis (see Tafalla et al. 2004 for further details). By fitting the radial profiles of $1.2 \mathrm{~mm}$ continuum emission, we find density profiles very close to those expected for isothermal spheres with central densities of $10^{5}$ and $2 \times 10^{5} \mathrm{~cm}^{-3}$ for L1498 and L1517B, respectively (see Alves et al. 2001 and Evans et al. 2001 for similar fits to other pre-stellar cores).

To derive the gas temperature profile, we use the $\mathrm{NH}_{3}$ emission, that we will see below traces well the inner core. From the combined analysis of the emission from the metastable $J, K=1,1$ and 2,2 levels, we derive constant temperature profiles for both cores with values close to $10 \mathrm{~K}$.

Finally, we derive core turbulent profiles using the linewidth of $\mathrm{NH}_{3}(1,1)$ complemented with other species. We subtract the (constant) thermal component and find a constant 

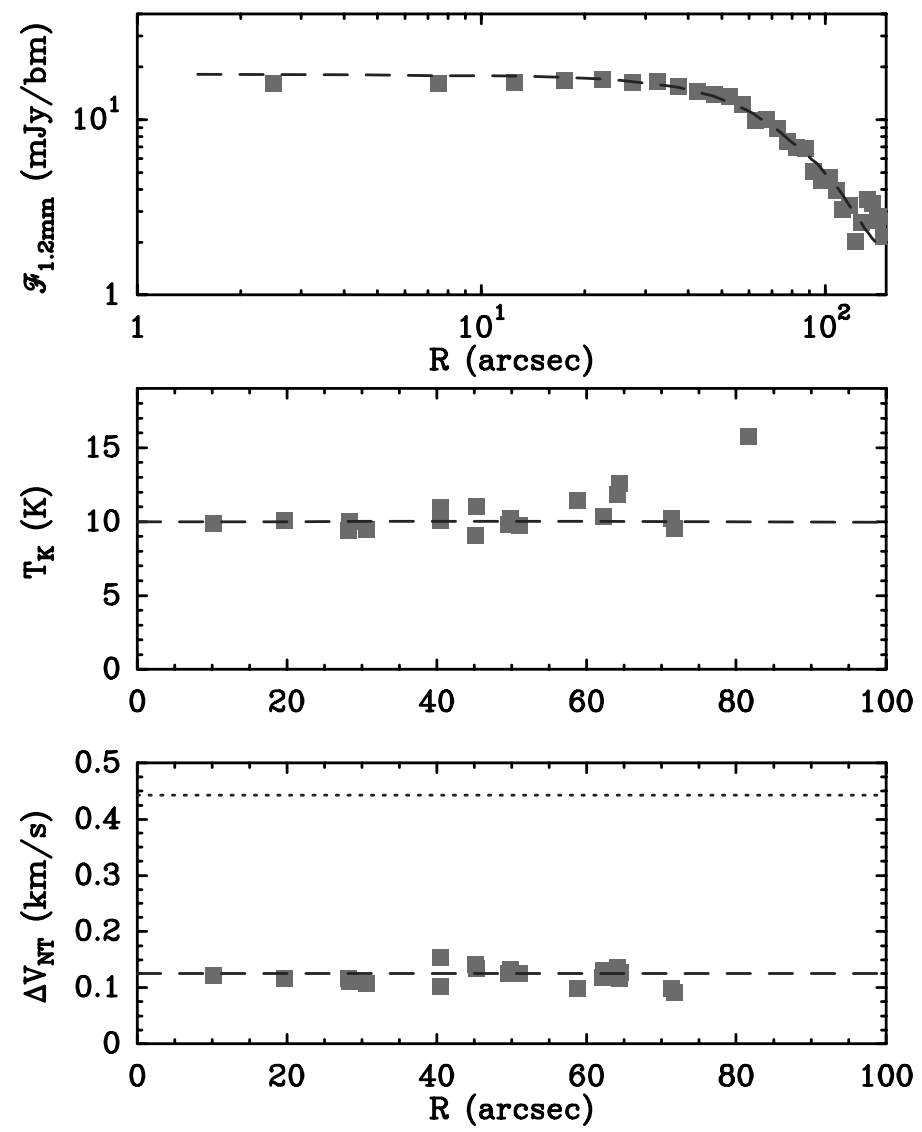

Figure 3. Determination of the physical parameters of L1498. Top: Radial profile of $1.2 \mathrm{~mm}$ continuum emission from the map of Figure 2 (squares) and prediction from the best fit density determination (dashed lines). Middle: $\mathrm{NH}_{3}$-derived gas kinetic temperature estimate (squares) and constant $10 \mathrm{~K}$ fit (dashed lines). Bottom: Non-thermal linewidth component derived from $\mathrm{NH}_{3}(1,1)$ spectra (squares) and constant component with $\mathrm{FWHM}=0.125 \mathrm{~km} \mathrm{~s}^{-1}$ (dashed lines). The dotted line indicates the expected value for a sonic component.

turbulent component of less than $1 / 3^{r d}$ the speed of sound. Such a low level of turbulence would seem problematic for current turbulent models of star formation.

\subsection{Chemical Structure of L1498 and L1517B}

Once the physical structure of each core has been determined, the only free parameter left to fit the observed emission of a given species is the radial distribution of its abundance. To convert this distribution into a predicted line intensity, we use a Monte Carlo radiative transfer code that assumes spherical symmetry (Bernes 1979). For each observed transition, we require that the model fits both the radial distribution of integrated intensity (derived by averaging the data azimuthally) and the line spectrum observed toward the core center. When possible, we use the thin emission of a rare isotopologue (such as $\mathrm{C}^{18} \mathrm{O}$ and $\mathrm{C}^{34} \mathrm{~S}$ ) to determine the abundance of the major species (in this case $\mathrm{CO}$ and CS). For most molecules we have observed two or more transitions, thus their abundance profile is over-determined by the data.

In a meeting like this one is important to emphasize that the analysis presented here can only be carried out if certain molecular parameters have been previously determined. 
Among these parameters, the collision rates are critical because they regulate the excitation of the levels and therefore the relation between model abundance and predicted intensity. In fact, the availability of collision rates with $\mathrm{H}_{2}$ or $\mathrm{He}$ was a main criterion for selecting the species observed in this survey. Another important molecular parameter needed to fit the narrow lines observed in L1498 and L1517B is the frequency of each transition. For our modelling, accuracies of $10 \mathrm{kHz}$ are required, and although this level is now easily achieved in the laboratory, not all important molecular transitions have yet been measured to this precision; accurate rest frequencies of molecular ions are especially critical. For our work, we have used the most recent frequency determinations from the CDMS, Gottlieb and collaborators, Dore and collaborators, and the JPL catalog.

To illustrate the process of deriving abundance profiles from the data, we present in Figure 4 (top) a sample of integrated intensity maps for the L1498 core. The three panels in the left column show centrally concentrated distributions corresponding to the $1.2 \mathrm{~mm}$ continuum, $\mathrm{N}_{2} \mathrm{H}^{+}(1-0)$, and $\mathrm{NH}_{3}(1,1)$. The rest of the maps, on the other hand, show ring-like distributions with a relative minimum at the dust peak. These ring-like distributions are quite fragmented and slightly different for each molecule, although there are systematic features, such as a brighter peak to the southeast.

When we convert the above maps into radial profiles of integrated intensity and attempt to fit them with different abundance profiles, we obtain the results shown in the bottom part of Figure 4. For each molecule, this figure shows two model predictions: a constant abundance model chosen to fit the emission in the outer core (dashed lines) and a bestfit model (solid lines). As the figure shows, the $\mathrm{N}_{2} \mathrm{H}^{+}$emission is fit reasonably well by the constant abundance model, while the observed $\mathrm{NH}_{3}$ emission is more centrally concentrated than predicted by a constant abundance model. This species, therefore, requires a significant enhancement of abundance toward the core center.

In agreement with the expectation from the ring-shaped maps, the rest of the molecules cannot be fit with a constant abundance model, as these models clearly overestimate the central intensity by a factor of 2 or more when forced to fit the outer core emission. Only using a sharp central abundance drop can both the outer and inner emission be simultaneously fit. For this reason, we have chosen for our best-fit models simple step functions with close to zero abundance toward the center. From the quality of the fit, we conclude that the data are consistent with a (close to) total absence of certain molecules at the core center.

Although not shown here for lack of space, the abundance results for L1517B are very similar to those for L1498 (a full account of the analysis will be presented in Tafalla et al. 2005 , in preparation). The outer abundances for both cores are in fact rather close, and for most species they agree within a factor of 2 ; this suggests that both objects have contracted from gas having similar chemical compositions. The size of the central hole, on the other hand, is different in the two cores. L1517B presents significantly smaller central molecular holes (about 50\% smaller than L1498), which may be related to the more concentrated gas distribution found in this core.

\subsection{Consequences for Dense Core Studies}

L1498 and L1517B seem in every aspect typical pre-stellar cores. More restricted studies of other cores by different authors, such as those presented in this meeting in the posters of Butner et al., Buckle et al., Friesen et al., Zinchenko et al., show molecular abundance patterns that are very similar to those found in L1498 and L1517B; it seems therefore natural to assume that the abundance profiles of L1498 and L1517B are representative of the pre-stellar core population as a whole (but see below for exceptions). Thus, despite 

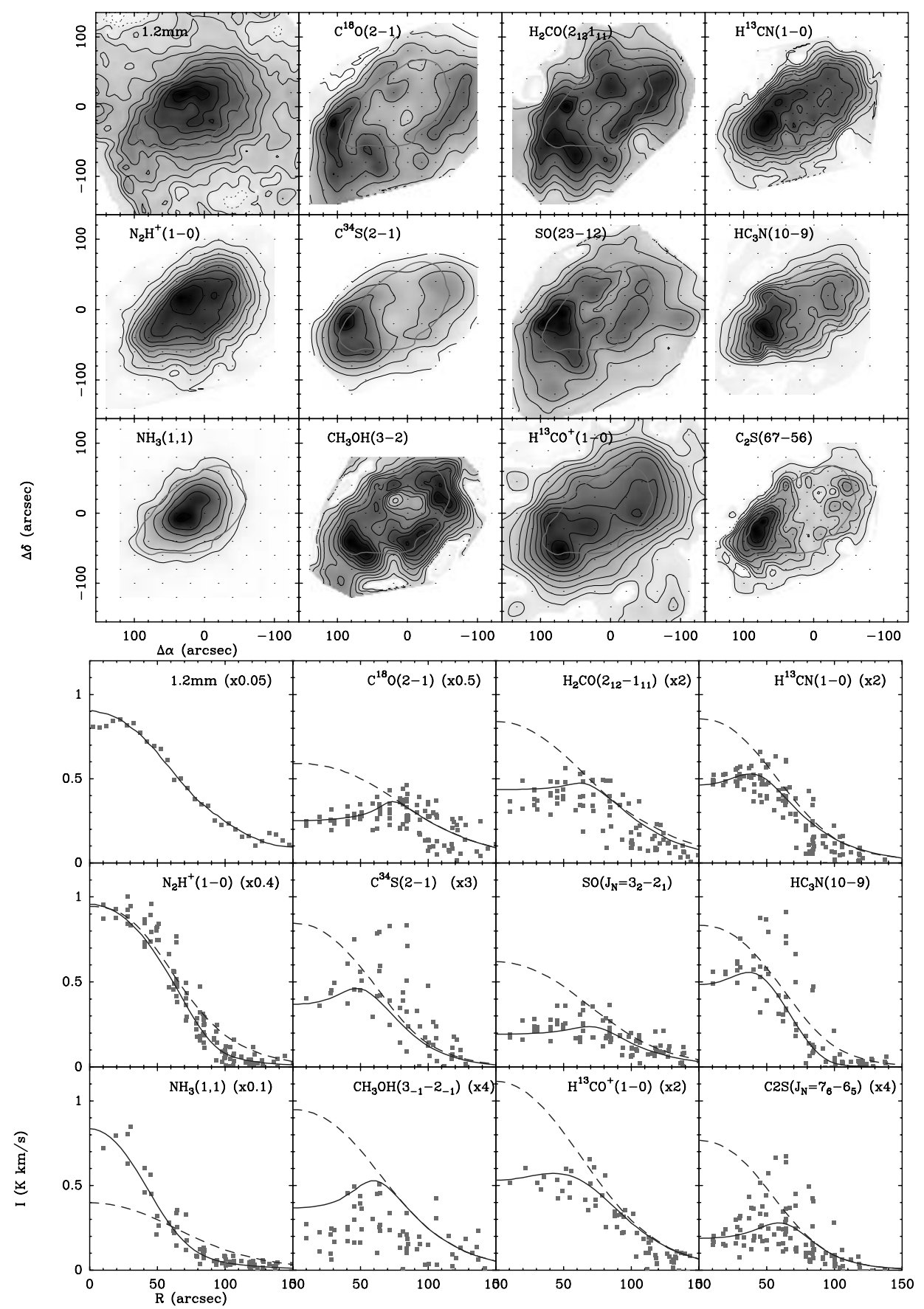

Figure 4. Partial results of the molecular survey toward L1498. Top: Maps of $1.2 \mathrm{~mm}$ continuum and molecular lines for a sample of transitions. The three panels in the leftmost column contain centrally concentrated emission maps, while the rest of the panels show ring-shaped distributions. Bottom: Radial profiles of emission derived from the maps in the top (squares) together with the emission predictions from two Monte Carlo models. The dashed lines are the predictions from constant abundance models set to fit the outer core emission, while the solid lines are predictions from the best-fit models. 
their expected simplicity, the observations force us to conclude that pre-stellar cores must have a strongly differentiated chemical composition.

If chemical inhomogeneities are part of the initial conditions of star formation, they need to be considered seriously when sampling star-forming gas with molecular tracers. The bottom panels in Figure 4, in particular, show that many molecular maps of a core may reflect more its chemical composition than its physical structure, and illustrate the danger in attributing emission peaks of even thin lines like $\mathrm{C}^{34} \mathrm{~S}(2-1)$ to actual core sub-structure. This analysis demonstrates that one needs to be careful when choosing dense gas tracers, and that $\mathrm{N}_{2} \mathrm{H}^{+}$and $\mathrm{NH}_{3}$ are two of the best choices. These species, together with their isotopologues and the $\mathrm{H}_{2} \mathrm{D}^{+}$ion recently detected by Caselli et al . (2003) in the pre-stellar core L1544, are probably all the molecular tracers left to study the conditions in the inner core (even $\mathrm{N}_{2} \mathrm{H}^{+}$may deplete at high densities, see Bergin et al. 2002 and Pagani et al. 2005). Posters in this meeting by Crapsi et al. and Vastel et al. show the use of these tracers to study the central conditions of pre-stellar cores.

The modelling of pre-stellar core chemistry is the subject of several contributions in this meeting (talks by Shematovich and Roberts, and posters by Aikawa et al., Rawlings, Lee et al., Walmsley et al.), so I refer to them for further details. The only point worth emphasizing here is that the unique behavior of species like $\mathrm{N}_{2} \mathrm{H}^{+}$and $\mathrm{NH}_{3}$ strongly suggests that their survival in the gas phase results from a low binding energy of $\mathrm{N}_{2}$, as initially suggested by Bergin \& Langer (1997) and Charnley (1997). However, similar binding energies for $\mathrm{N}_{2}$ and $\mathrm{CO}$ have been recently measured by Öberg et al. (2005), a result that has been used to claim that other mechanisms, like a low sticking coefficient for molecular or atomic $\mathrm{N}$, may be needed to explain the behavior of $\mathrm{N}_{2} \mathrm{H}^{+}$and $\mathrm{NH}_{3}$ (Flower et al. 2005). Further work in this topic is clearly needed if we are to understand and use to our advantage the peculiar chemistry of these two important dense gas tracers.

\section{Searching for Young Pre-Stellar Cores}

If we observe pre-stellar cores selected from $\mathrm{NH}_{3}$ surveys (like those from Myers and collaborators), we find that molecular depletion is the norm without exception. This systematic trend suggests that $\mathrm{NH}_{3}$-selected cores are significantly advanced in their process of gas contraction from the ambient cloud, and that there must exist a population of younger cores that are their precursors. Identifying such a population of cores is interesting not only because of their chemical properties, but because its members should constitute a missing link between cloud and core conditions, and they will therefore provide useful clues on the (mysterious) physical process that drives core condensation out of ambient material.

In the search for young pre-stellar cores, we can take advantage of their expected chemical properties. One of them is a lower degree of CO freeze out at the center, which should make a young pre-stellar core appear centrally concentrated in a $\mathrm{C}^{18} \mathrm{O}$ map. Another expected property is a low abundance of late-time species like $\mathrm{N}_{2} \mathrm{H}^{+}$and $\mathrm{NH}_{3}$, which will translate into weak emission in any line of these tracers. To relate these properties, we define the intensity ratio

$$
R=\frac{I\left[\mathrm{C}^{18} \mathrm{O}(1-0)\right]}{I\left[\mathrm{~N}_{2} \mathrm{H}^{+}(1-0)\right]},
$$

where the intensities are evaluated at the core center. This ratio is an easily measurable quantity, as both lines lie in the $3 \mathrm{~mm}$ wavelength band and can therefore be observed with similar angular resolution. Young cores are expected to have relatively large central $\mathrm{C}^{18} \mathrm{O}(1-0)$ emission together with weak $\mathrm{N}_{2} \mathrm{H}^{+}(1-0)$ lines, so they are expected to have 


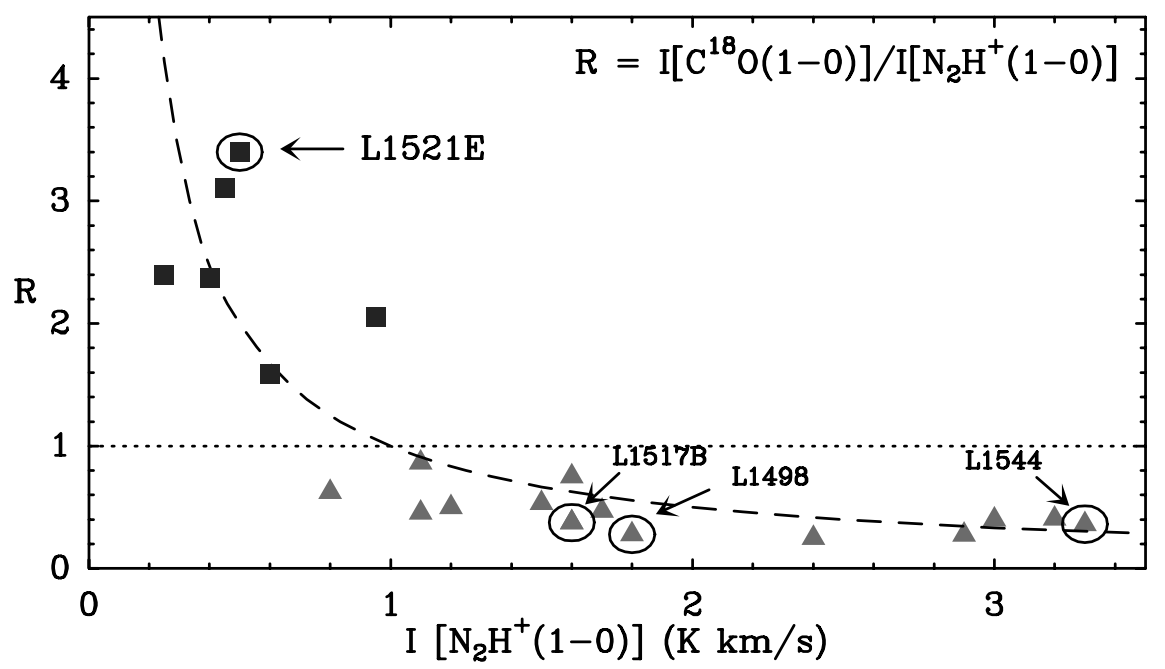

Figure 5. $\mathrm{R}\left(=\mathrm{I}\left[\mathrm{C}^{18} \mathrm{O}(1-0)\right] / \mathrm{I}\left[\mathrm{N}_{2} \mathrm{H}^{+}(1-0)\right]\right)$ as function of $\mathrm{I}\left[\mathrm{N}_{2} \mathrm{H}^{+}(1-0)\right]$ for two samples of cores. The triangles are "classical" starless cores selected for their easily detectable $\mathrm{NH}_{3}$ emission, while the squares are starless cores selected for their weak $\mathrm{NH}_{3}$ emission. The dotted horizontal line marks the expected approximate boundary between cores with and without $\mathrm{C}^{18} \mathrm{O}$ freeze out, and the dashed line is the prediction from a toy model of core chemical evolution. Note how L1521E stands out among the young core candidates $(\mathrm{R}>1)$.

relatively large values of the $R$ parameter. Conversely, old cores are expected to have relatively weak central $\mathrm{C}^{18} \mathrm{O}(1-0)$ emission and strong $\mathrm{N}_{2} \mathrm{H}^{+}(1-0)$ lines, so they should be characterized by relatively low values of $R$. The approximate boundary between these two behaviors can be calculated using the ratio predicted by our Monte Carlo model for cores like L1498 and L1517B assuming constant abundances for the two species. In this way, we find the expected separation between young and old cores near the value $R=1$.

As mentioned before, searches for young cores using $\mathrm{NH}_{3}$-selected candidates seem doomed to fail. This is illustrated by the triangles in Figure 5, which depict a survey of that type of objects carried out with the FCRAO telescope. In this figure, all selected objects lie below the $R=1$ line, including (not surprisingly) L1498 and L1517B studied before. The objects from this survey probably span a range of ages, as suggested by the range of $\mathrm{N}_{2} \mathrm{H}^{+}(1-0)$ intensities that starts near 1 and ends past 3 with evolved cores like L1544 (e.g., Crapsi et al. 2005). However, they seem to be missing the youngest cores.

To identify candidates as young starless cores we need to include cores having weak $\mathrm{NH}_{3}$ emission, and this has been done selecting sources from the survey of Suzuki et al. (1992) and from a survey of the L1521 filament in Taurus using the FCRAO telescope. These cores finally fill the region in the plot expected for young cores, as they have low $\mathrm{N}_{2} \mathrm{H}^{+}(1-0)$ intensities together with large $R$ values. Among these objects, L1521E has the largest $R$ ratio $(=3.4)$, and therefore appears as the best candidate for a young starless core. Previous suggestions of this core being extremely young have been made by Suzuki et al. (1992) and Hirota et al. (2002).

Given the unusual characteristics of L1521E, we have carried out a molecular survey of this core in a similar manner as we have studied L1498 and L1517B. From a preliminary analysis of these data, we conclude that it has no significant CO or CS central depletion (Tafalla \& Santiago 2004), and that its $\mathrm{N}_{2} \mathrm{H}^{+}$and $\mathrm{NH}_{3}$ abundances are 8 and 20 times lower, respectively, than L1498 and L1517B. These characteristics truly classify L1521E as a chemically young pre-stellar core, and therefore suggest that this object 
has contracted from the ambient cloud to its observed state rather recently. Surprisingly, however, L1521E has a central density of $10^{5} \mathrm{~cm}^{-3}$, which is very similar to that found in L1498 and L1517B. This high density contradicts the expectation that a young core should be less dense, and suggests that L1521E may have contracted faster than the others (also Aikawa et al. 2005). Clearly, more work is needed to clarify the origin of this group of starless cores, and recent studies of similar systems are encouraging (Hirota et al. 2004, Morata et al. 2005). The poster by Hirota and Yamamoto in this meeting provides recent results on this topic.

\section{Acknowledgements}

I thank the organizers for their invitation and for a highly enjoyable and productive meeting. Part of the work presented here is the result of an ongoing collaboration with Joaquín Santiago, Phil Myers, Paola Caselli, Malcolm Walmsley, Claudia Comito, and Antonio Crapsi. I thank them for help and discussions on pre-stellar cores over the last several years.

\section{References}

Adams, F.C. \& Myers, P.C. 2001, Ap. J. 553, 744

Aikawa, Y., Herbst, E., Roberts, H., \& Caselli, P. 2005, Ap. J. 620, 330

Alves, J., Lada, C.J., \& Lada, E.A. 2001, Nature 409, 159

Benson, P.J. \& Myers, P.C. 1989, Ap. J. Suppl. 71, 89

Bergin, E.A. \& Langer, W.D. 1997, Ap. J. 486, 316

Bergin, E.A., Alves, J., Huard, T., \& Lada, C.J. 2002, Ap. J. 570, L101

Bernes, C. 1979, A\& $A$ 73, 67

Caselli, P., Walmsley, C.M., Tafalla, M., Dore, L. \& Myers, P.C. 1999, Ap. J. 523, L165

Caselli, P., van der Tak, F.F.S., Ceccarelli, C., \& Bacmann, A. 2003, A\&A 403, L37

Charnley, S.B. 1997, MNRAS 291, 455

Crapsi, A., Caselli, P., Walmsley, C.M., Myers, P.C., Tafalla, M., Lee, C.W., \& Bourke, T.L. 2005, Ap. J. 619, 379

Evans, N.J., II, Rawlings, J.M.C., Shirley, Y.L., \& Mundy, L.G. 2001 Ap. J. 557, 193

Flower, D.R., Pineau Des Forêts, G., \& Walmsley, C.M.2005 A\&A 436, 933

Hirota, T., Ito, T., \& Yamamoto, S. 2002 Ap. J. 565, 359

Hirota, T., Maezawa, H., \& Yamamoto, S. 2004 Ap. J. 617, 399

Kramer, C., Alves, J., Lada, C.J., Lada, E.A., Sievers, A., Ungerechts \& Walmsley, C.M. 1999, A\&A 342,257

Kuiper, T.B.H., Langer, W.D., \& Velusamy, T. 1996, Ap. J. 468, 761

Mac Low, M.-M. \& Klessen, R.S. 2004, Rev. Mod. Phys. 76, 125

Morata, O., Girart, J.M., \& Estalella, R. 2005 A\&A 435, 113

Mouschovias, T.C., \& Ciolek, G.E. 1999, in C.J. Lada and N.D. Kylafis (eds.), The Origin of Stars and Planetary Systems (Dordrecht: Kluwer), p. 305

Öberg, K.I., van Broekhuizen, F., Fraser, H.J., Bisschop, S.E., van Dishoeck, E.F., \& Schlemmer, S. 2005, A\&A 621, L33

Pagani, L., Pardo, J.-R., Apponi, A.J., Bacmann, A., \& Cabrit, S. 2005, A\&\&A 429, 181

Shu, F.H., Adams, F.C., \& Lizano S. 1987, ARAA 25, 23

Suzuki, H., Yamamoto, S., Ohishi, M., Kaifu, N., Ishikawa, S.-I., Hirahara, Y., \& Takano, S. 1992, Ap. J. 392, 551

Tafalla, M., Myers, P.C., Caselli, P., \& Walmsley, C.M. 2004, A\& A 416, 191

Tafalla, M. \& Santiago, J. 2004, A\&A 414, L53

Ward-Thompson, D., Motte, F., \& André, P. 1999, A\&\&A 305, 143

Watson, W.D. \& Salpeter, E.E. 1972, Ap. J. 175, 659

Willacy, K., Langer, W.D., \& Velusamy, T. 1998, Ap. J. 507, L171

Zhou, S., Wu, Y., Evans, N.J.II, Fuller, G.A., \& Myers, P.C. 1989, Ap. J. 346, 168 


\section{Discussion}

GEBALLE: You stated at the beginning of your talk that some pre-stellar cores display outflows. How is it possible without a star (or protostar) in the core and such objects being "pre-stellar" cores?

TAFALLA: I'm afraid there is some misunderstanding here. I mentioned that stars forming in cores like L1498 and L1517B will develop outflows, but of course they need to form a protostar first. As far as we know (and we have looked), these two cores are starless and therefore have no outflow.

JOHnstone: We should be careful using terms like "young" when the evolution of a core is unknown. How a core forms and evolves is still poorly known and perhaps we should be clearer in the terminology using expressions like "early differentiation" or the like. Still, the ratio relation clearly shows an age direction with lower $R$ 's being more evolved cores.

TAFALLA: I agree that we have to be careful connecting the chemical and physical evolution of cores. At this point, when we say "young" we usually mean having low chemical processing according to models. The main unknown connecting physical and chemical evolution is whether all cores start with similar chemical composition, so chemical differences can be interpreted as differences either in age or in speed of contraction.

PAPAdopoulos: A note rather than a question. Quiescent pre-stellar cores are not a problem for the turbulent-driven star formation, since from the first proposition by Larson to the latest work by Krumholz and McKee, such quiescent cores/places in the ISM are the ones predicted to detach from the turbulent hierarchy and settle to a star-formationcapable phase with thermal energy dominating over the turbulent one.

TAFAlla: That is exactly why identifying young cores is so interesting for testing core contraction models. If cores form by the dissipation of turbulence, we should find a systematic decrease in linewidth with age.

FALGARONE: What is the contribution of the intrinsic bistability of steady-state chemistry in dense cores to the observed chemical differentiation?

TAFAlla: Not being an expert on chemical models, I am not the best person to answer this question. As far as I know, there is no analysis of bistability that takes into account molecular freeze out and dust chemistry. Probably someone should look into this problem.

HenNing: If you assume a sphere, you will get a sphere. How good is this assumption? Actually, we demonstrated that one better should do a 3D analysis

TAFAlla: We especially chose our cores for their simple geometry and close to round shape in the plane of the sky. Of course we cannot constrain the line of sight dimension, so we assume that it will not be too different from what we observe in the plane of the sky. By observing multiple cores, we expect to minimize the chance of being confused by objects having an anomalous line-of-sight dimension. 